\title{
Impulsive surfaces on dynamical systems
}

\author{
E. M. Bonotto ${ }^{\mathrm{a}, 1}$, M. C. Bortolan ${ }^{\mathrm{b}}$, T. Caraballo ${ }^{*, c, 2}$, R. Collegari ${ }^{\mathrm{d}, 3}$ \\ ${ }^{a}$ Instituto de Ciências Matemáticas e de Computação, Universidade de São Paulo - \\ Campus de São Carlos, 13560-970, São Carlos SP, Brazil. \\ ${ }^{b}$ Departamento de Matemática, Universidade Federal de Santa Catarina, Campus Trindade, \\ 88040-900, Florianópolis, Brazil. \\ ${ }^{c}$ Dpto. Ecuaciones Diferenciales y Análisis Numérico, Universidad de Sevilla, Apdo. de \\ Correos 1160, 41080 - Sevilla, Spain. \\ ${ }^{d}$ Instituto de Ciências Matemáticas e de Computação, Universidade de São Paulo - \\ Campus de São Carlos, 13560-970, São Carlos SP, Brazil.
}

\begin{abstract}
This work is devoted to the construction of impulsive sets in $\mathbb{R}^{n}$. In the literature, there are many examples of impulsive dynamical systems whose impulsive sets are chosen in an abstract way, and in this paper we present sufficient conditions to characterize impulsive sets in $\mathbb{R}^{n}$ which satisfy some "tube conditions" and ensure a good behavior of the flow. Moreover, we present some examples to illustrate the theoretical results.
\end{abstract}

Key words: Impulsive sets; hypersurfaces; dynamical systems.

2010 MSC: Primary 53A05; Secondary 34A37.

\section{Introduction}

Oftentimes it is necessary to cause an abrupt change in the evolution of a real model in order to avoid an inconsistent result. One of the theories used to study such systems is the theory of impulsive differential equations - the reader may consult [13] for more details and [5], [6], [9], [14], [15] for some applications.

\footnotetext{
* Corresponding author

Email addresses: ebonotto@icmc.usp.br (E. M. Bonotto), m.bortolan@ufsc.br (M. C. Bortolan), caraball@us.es (T. Caraballo), collegari@gmail.com (R. Collegari)

${ }^{1}$ Partially supported by FAPESP grant 2014/25970-5 and CNPq grant 307317/2013-7.

${ }^{2}$ Partially supported by FEDER and Ministerio de Economía y Competitividad (Spain) under grant MTM2015-63723-P, and Consejería de Innovación, Ciencia y Empresa (Junta de Andalucía) under Proyecto de Excelencia P12-FQM-1492.

${ }^{3}$ Partially supported by FAPESP grants 2013/23933-2 and 2014/20691-0.
} 
Recently there has been an interest in the theory of impulsive dynamical systems (IDS, for short) since it possesses a richer dynamics than the continuous case. In this new framework, the impulsive set is in the phase space and the impulses vary on time, and are not pre-assigned. The reader is referred to $[2],[3],[1],[4],[7],[8],[10],[12]$ for more details. This impulsive set plays an important role to control the evolution of the system. For instance, in the study of an impulsive predator-prey model presented in [15], when the density of the prey hits a level which is the threshold with slight damage (this level is the impulsive set), then there are released its natural predators in order to control the environment (the addition of predators is represented by an impulse function). One of the first and crucial conditions we must have is that the impulsive set needs to be, in some sense, transversal to the flow, and moreover, in order to obtain important topological properties for impulsive systems, it also needs to satisfy what we call "tube conditions", which is presented in Definition 4. Due to the difficulties to verify the tube condition for impulsive sets in general spaces, some examples in the literature consider abstract impulsive sets satisfying such condition.

In this work, we present sufficient conditions for a class of impulsive sets to satisfy the tube condition in $n$-dimensional spaces, which is very important since we have plenty of applications in finite dimensional spaces. Some examples are included at the end of the paper.

\section{Preliminaries}

We begin our study by briefly recalling the theory of impulsive dynamical systems. Let $(X, d)$ be a metric space with metric $d, \mathbb{R}_{+}$be the set of nonnegative real numbers and $\mathbb{N}=\{1,2,3, \ldots\}$ be the set of natural numbers. A semigroup in $X$ is a family of functions $\{\pi(t): t \geqslant 0\}$ from $X$ to $X$, indexed on $\mathbb{R}_{+}$, satisfying the following conditions:

i) $\pi(0) x=x$ for all $x \in X$;

ii) $\pi(t+s)=\pi(t) \pi(s)$ for all $t, s \in \mathbb{R}_{+}$; 
iii) the map $\mathbb{R}_{+} \times X \ni(t, x) \mapsto \pi(t) x$ is continuous.

Let $\{\pi(t): t \geqslant 0\}$ be a semigroup in $X$. For each $D \subseteq X$ and $J \subseteq \mathbb{R}_{+}$we define $F(D, J)=\bigcup_{t \in J} \pi(t)^{-1}(D)$. A point $x \in X$ is called an initial point if $F(x, t)=\varnothing$ for all $t>0$.

Definition 1. An impulsive dynamical system (IDS, for short) $(X, \pi, M, I)$ consists of a semigroup $\{\pi(t): t \geqslant 0\}$ on a metric space $(X, d)$, a nonempty closed subset $M \subseteq X$ such that for every $x \in M$ there exists $\epsilon_{x}>0$ such that

$$
F\left(x,\left(0, \epsilon_{x}\right)\right) \cap M=\varnothing \quad \text { and } \bigcup_{t \in\left(0, \epsilon_{x}\right)}\{\pi(t) x\} \cap M=\varnothing,
$$

and a continuous function $I: M \rightarrow X$ (its role will be specified later). The set $M$ is called the impulsive set and the function $I$ is called impulsive function.

Remark 2. Condition (1) means that the flow of the semigroup $\{\pi(t): t \geqslant 0\}$ is, in some sense, transversal to $M$ at any point of $M$.

Thanks to condition (1) we may define the function $\phi: X \rightarrow(0,+\infty]$ by

$$
\phi(x)= \begin{cases}s, & \text { if } \pi(s) x \in M \text { and } \pi(t) x \notin M \text { for } 0<t<s, \\ +\infty, & \text { if } \pi(t) x \notin M \text { for all } t>0 .\end{cases}
$$

If $\pi(t) x \in M$ for some $t>0$, then the value $\phi(x)$ represents the smallest positive time such that the trajectory of $x$ meets $M$. In this case, we say that the point $\pi(\phi(x)) x$ is the impulsive point of $x$.

The impulsive positive trajectory of $x \in X$ by the IDS $(X, \pi, M, I)$ is a map $\tilde{\pi}(\cdot) x$ defined in an interval $J_{x} \subseteq \mathbb{R}_{+}, 0 \in J_{x}$, with values in $X$ given inductively by the following rule: if $\phi(x)=+\infty$ then $\tilde{\pi}(t) x=\pi(t) x$ for all $t \in \mathbb{R}_{+}$. However, if $\phi(x)<+\infty$ then we denote $x=x_{0}^{+}$and define $\tilde{\pi}(\cdot) x$ on $\left[0, \phi\left(x_{0}^{+}\right)\right]$by

$$
\tilde{\pi}(t) x=\left\{\begin{array}{lll}
\pi(t) x_{0}^{+}, & \text {if } & 0 \leqslant t<\phi\left(x_{0}^{+}\right), \\
I\left(\pi\left(\phi\left(x_{0}^{+}\right)\right) x_{0}^{+}\right), & \text {if } & t=\phi\left(x_{0}^{+}\right) .
\end{array}\right.
$$


Now let $s_{0}=\phi\left(x_{0}^{+}\right), x_{1}=\pi\left(s_{0}\right) x_{0}^{+}$and $x_{1}^{+}=I\left(\pi\left(s_{0}\right) x_{0}^{+}\right)$. In this case $s_{0}<+\infty$ and the process can go on, but now starting at $x_{1}^{+}$. This process ends after a finite number of steps if $\phi\left(x_{n}^{+}\right)=+\infty$ for some $n \in \mathbb{N} \cup\{0\}$, or it may proceed indefinitely, if $\phi\left(x_{n}^{+}\right)<+\infty$ for all $n \in \mathbb{N}$ and, in this case, $\tilde{\pi}(\cdot) x$ is defined in the interval $[0, T(x))$, where $T(x)=\sum_{i=0}^{\infty} s_{i}$. The reader may see [3], [1], [7], [8], [12] for more details.

Next, we present the concepts of tube and STC which allow us to obtain important topological properties for impulsive systems.

Definition 3. Let $\{\pi(t): t \geqslant 0\}$ be a semigroup on $X$. A closed set $S$ containing $x \in X$ is called a section through $x$ if there exists $\lambda>0$ and a closed subset $L$ of $X$ such that:

i) $F(L, \lambda)=S$;

ii) $F(L,[0,2 \lambda])$ contains a neighborhood of $x$;

iii) $F(L, \nu) \cap F(L, \zeta)=\emptyset$, if $0 \leqslant \nu<\zeta \leqslant 2 \lambda$.

The set $F(L,[0,2 \lambda])$ is called a $\lambda$-tube (or simply tube) and the set $L$ is called a bar.

Definition 4. Let $(X, \pi, M, I)$ be an IDS. We say that a point $x \in M$ satisfies the strong tube condition (STC), if there exists a section $S$ through $x$ such that $S=F(L,[0,2 \lambda]) \cap M$. We say that $M$ satisfies $S T C$ if each point $x \in M$ satisfies $S T C$.

\section{The Main Results}

Let $f: \mathbb{R}^{n} \rightarrow \mathbb{R}^{n}$ be a function such that the autonomous differential equation

$$
\dot{x}=f(x), \quad x \in \mathbb{R}^{n},
$$

defines a dynamical system in $\mathbb{R}^{n}$. In particular, we also assume that equation (3) satisfies conditions for the existence, uniqueness and extendability to the 
whole real line of its solutions for all initial points in $\mathbb{R}^{n}$ as well as the continuous dependence on initial conditions.

Let $\left(\mathbb{R}^{n}, \pi\right)$ be the dynamical system generated by system (3). Thus $\pi$ is a group and $F(x, t)=\pi^{-1}(t) x=\pi(-t) x$ for all $x \in \mathbb{R}^{n}$ and $t \geqslant 0$. Moreover, the map $\mathbb{R} \times \mathbb{R}^{n} \ni(t, x) \mapsto \pi(t) x$ is continuous. In the sequel, we present a class of impulsive dynamical systems $\left(\mathbb{R}^{n}, \pi, M, I\right)$ associated to $\left(\mathbb{R}^{n}, \pi\right)$ such that $M$ satisfies STC. Since the impulsive set needs to be closed and, in some sense, transversal to the flow, from now on we will always assume that $M \subset \mathbb{R}^{n}$ is a hypersurface in $\mathbb{R}^{n}$ of class $C^{k}$ (a closed $(n-1)$-dimensional submanifold of $\mathbb{R}^{n}$ of class $\left.C^{k}\right), k \geqslant 1$, satisfying the following transversality condition:

$$
\text { for each } p \in M \text { we have }\left\langle\vec{n}_{p}, f(p)\right\rangle \neq 0 \text {, }
$$

where $\vec{n}_{p}$ denotes the normal vector of $M$ at $p$, and $\langle\cdot, \cdot\rangle$ is the scalar product in $\mathbb{R}^{n}$.

Let $\mathcal{S} \subset \mathbb{R}^{n}$ be a manifold in $\mathbb{R}^{n}$. A differentiable map $g: \mathcal{S} \rightarrow \mathbb{R}$ is called a submersion at a point $p \in \mathcal{S}$ if the differential map $\mathrm{d} g(p): \mathbb{R}^{n} \rightarrow \mathbb{R}$ is a surjective linear map and $g$ is called a submersion if it is a submersion at each point $p \in \mathcal{S}$. A point $c \in \mathbb{R}$ is a regular value of $g$ if for each point $p \in g^{-1}(c)$, $g$ is a submersion at $p$. Next, we present an auxiliary result and the reader is referred to [11] for more details.

Lemma 5. Let $\mathcal{S} \subset \mathbb{R}^{n}$ be a manifold of class $C^{k}$ in $\mathbb{R}^{n}$. Then for each $p \in \mathcal{S}$, there exist an open set $V \subset \mathbb{R}^{n}, p \in V$, and a submersion $g: V \rightarrow \mathbb{R}$ of class $C^{k}$ such that $\mathcal{S} \cap V=g^{-1}(0)$. Moreover, $T_{p} \mathcal{S}=\operatorname{ker} \mathrm{d} g(p)$ and $\mathcal{S} \cap V$ may be assumed to be connected.

Using the previous lemma and condition (T) we obtain the following result.

Lemma 6. Let $p \in M, V \subset \mathbb{R}^{n}$ be an open set with $p \in V$ and $g: V \rightarrow \mathbb{R}$ be a submersion of class $C^{k}$ such that $M \cap V=g^{-1}(0)$. Then $f(p) \notin \operatorname{ker} \mathrm{d} g(p)$.

In the sequel, we show that hypersurfaces in $\mathbb{R}^{n}$ satisfying $(\mathbf{T})$ are impulsive sets satisfying STC. Lemma 7 shows the condition of transversality of the flow and Theorem 9 gives us the STC. 
Lemma 7. Given $x \in M$, there exists $\epsilon_{x}>0$ such that $F\left(x,\left(0, \epsilon_{x}\right)\right) \cap M=\varnothing$ and $\pi\left(\left(0, \epsilon_{x}\right)\right) x \cap M=\varnothing$.

Proof. Arguing by contradiction, suppose that there exists a sequence $\left\{t_{n}\right\}_{n \in \mathbb{N}} \subset \mathbb{R}_{+}, t_{n} \stackrel{n \rightarrow+\infty}{\longrightarrow} 0$, such that $\pi\left(t_{n}\right) x \in M, n \in \mathbb{N}$. By Lemma 5 , there are an open set $V \subset \mathbb{R}^{n}$, with $x \in V$, and a submersion $\beta: V \rightarrow \mathbb{R}$ of class $C^{k}, k \geqslant 1$, such that $V \cap M=\beta^{-1}(0)$. We can assume that there exists $\lambda>0$ such that $\pi([0, \lambda]) x \subset V$ and $t_{n}<\lambda$, for all $n \in \mathbb{N}$. Defining $h:[0, \lambda] \rightarrow \mathbb{R}$ by $h(t)=\beta(\pi(t) x)$, we have $h(0)=h\left(t_{n}\right)=0$ for all $n \in \mathbb{N}$ and, by the Rolle Theorem, there exists $\tau_{n} \in\left(0, t_{n}\right)$ such that

$$
h^{\prime}\left(\tau_{n}\right)=\left\langle\nabla \beta\left(\pi\left(\tau_{n}\right) x\right), f\left(\pi\left(\tau_{n}\right) x\right)\right\rangle=0, n \in \mathbb{N} .
$$

As $n \rightarrow+\infty$, we obtain $\langle\nabla \beta(x), f(x)\rangle=0$, which contradicts Lemma 6 .

Analogously, if there exists a sequence $\left\{t_{n}\right\}_{n \in \mathbb{N}} \subset \mathbb{R}_{+}, t_{n} \stackrel{n \rightarrow+\infty}{\longrightarrow} 0$, such that $F\left(x, t_{n}\right) \cap M \neq \emptyset$ for all $n \in \mathbb{N}$, then we can also assume that there exists $\lambda>0$ such that $F(x,[0, \lambda]) \subset V$ and $t_{n}<\lambda$ for all $n \in \mathbb{N}$. For each $n \in \mathbb{N}$, let $y_{n} \in F\left(x, t_{n}\right) \cap M$ and define the mapping $h_{n}:\left[0, t_{n}\right] \rightarrow \mathbb{R}$ by $h_{n}(t)=\beta\left(\pi(t) y_{n}\right)$. Using again the Rolle Theorem we obtain $\tau_{n} \in\left(0, t_{n}\right)$ with $h_{n}^{\prime}\left(\tau_{n}\right)=0, n \in \mathbb{N}$, that yields $\langle\nabla \beta(x), f(x)\rangle=0$ which is a contradiction. Hence, the result is proved.

Lemma 8. Let $V \subset \mathbb{R}^{n}$ be an open set and $\beta: V \rightarrow \mathbb{R}$ be a submersion such that $\beta^{-1}(0)=V \cap M \neq \varnothing$. If $x \in V \cap M$ and $t>0$ are such that $\pi((0, t]) x \subset V \backslash M$ and $\pi([-t, 0)) x \subset V \backslash M$ then $\beta(\pi(t) x) \beta(\pi(-t) x)<0$.

Proof. Let $x \in V \cap M$ and $t>0$ satisfying the statement of the lemma. First, we note that $\beta(\pi(t) x) \beta(\pi(s) x)>0$ for all $0<s \leqslant t$. Indeed, if there exists $0<s<t$ such that $\beta(\pi(t) x) \beta(\pi(s) x)<0$, then one can obtain $\tau \in(s, t)$ such that $\beta(\pi(\tau) x)=0$, that is, $\pi(\tau) x \in M$ which is a contradiction. Analogously, we have $\beta(\pi(-t) x) \beta(\pi(-s) x)>0$ for all $0<s \leqslant t$. Now, suppose the opposite, i.e., that $\beta(\pi(t) x) \beta(\pi(-t) x)>0$. Then, either $\beta(\pi([-t, 0) \cup(0, t]) x)>0$ or $\beta(\pi([-t, 0) \cup(0, t]) x)<0$ which implies that $\langle\nabla \beta(x), f(x)\rangle=0$ and gives also a contradiction by Lemma 6 . 
Theorem 9. The set $M \subset \mathbb{R}^{n}$ is an impulsive set which satisfies $S T C$.

Proof. By Lemma 7 the closed set $M$ is an impulsive set. Now let us show that $M$ satisfies STC. Let $x \in M$ be given. By Lemma 5 there exist an open set $V \subset \mathbb{R}^{n}$ with $x \in V$ and a submersion $\beta: V \rightarrow \mathbb{R}$ such that $V \cap M=$ $\beta^{-1}(0)$ is connected. Using the same argument as in Lemma 7, we may obtain a compact set $S \subset V \cap M, x \in S$, and $\lambda>0$ be such that $\pi((0,2 \lambda]) S \subset V \backslash M$ and $F(S,(0,2 \lambda]) \subset V \backslash M$. Define $L=\pi(\lambda) S$. We are going to show that $F(L,[0,2 \lambda])$ is a $\lambda$-tube through $x$.

It is clear that $F(L, \lambda)=S$. Now, suppose by contradiction that $F(L,[0,2 \lambda])$ does not contain a neighborhood of $x$, that is, there exists a sequence $x_{n} \stackrel{n \rightarrow+\infty}{\longrightarrow} x$ such that $x_{n} \notin F(L,[0,2 \lambda])$ for all $n \in \mathbb{N}$. By Lemma 8 , there exists $0<s<\lambda$ such that $\beta(\pi(s) x) \beta(\pi(-s) x)<0$, consequently there is $n_{0} \in \mathbb{N}$ such that $\pi([-s, s]) x_{n} \subset V$ and $\beta\left(\pi(s) x_{n}\right) \beta\left(\pi(-s) x_{n}\right)<0$ for all $n \geq n_{0}$. Thus, for $n \geq n_{0}$, there is $\tau_{n} \in(-s, s)$ such that $\beta\left(\pi\left(\tau_{n}\right) x_{n}\right)=0$, that is, $\pi\left(\tau_{n}\right) x_{n} \in M$, which gives a contradiction. To finish the proof, let us show that $F(L, \nu) \cap F(L, \mu)=\emptyset, 0 \leqslant \nu<\mu \leqslant 2 \lambda$. Suppose, again by contradiction, that there exists $y \in F(L, \nu) \cap F(L, \mu)$ for some $0 \leqslant \nu<\mu \leqslant 2 \lambda$. Then $a=\pi(\nu-\lambda) y \in S$ and $b=\pi(\mu-\lambda) y \in S \subset M$. Consequently, $\pi(\mu-\nu) a=b \in M$ and this is a contradiction since $a \in S$ and $\pi((0,2 \lambda]) S \cap M=\varnothing$ as we have $\pi((0,2 \lambda]) S \subset V \backslash M$, which concludes the proof.

Corollary 10. Let $M \subset \mathbb{R}^{n}$ be a hypersurface in $\mathbb{R}^{n}$ satisfying $(\mathbf{T})$ and $I: M \rightarrow$ $\mathbb{R}^{n}$ be a continuous function. Then $\left(\mathbb{R}^{n}, \pi, M, I\right)$ is an IDS with $M$ satisfying STC.

Example 11. In [4, Example 3.2], the authors study the uniform stability of the system

$$
\left\{\begin{array}{l}
\dot{x}=-\alpha y+y z-x^{3} \\
\dot{y}=x-\beta x z-\gamma y^{3} \\
\dot{z}=\theta x y-z^{3}
\end{array}\right.
$$

subject to the impulsive condition $I: M \rightarrow \mathbb{R}^{3}$, where $\alpha, \beta, \gamma, \theta>0$, $\beta \alpha>1, M$ is an impulsive set and $I$ is an impulse function. The set $M$ 
was taken arbitrary and satisfying the condition STC. It is natural to ask for a concrete impulsive set satisfying STC. For instance, if $M=\{(x, y, z) \in$ $\left.\mathbb{R}^{3}: x^{2}+\alpha y^{2}+\frac{\alpha \beta-1}{\theta} z^{2}-r^{2}=0\right\}$, where $r>0$ is a constant, then $M$ is an impulsive set satisfying STC. In fact, let $g: \mathbb{R}^{3} \rightarrow \mathbb{R}$ be given by

$$
g(x, y, z)=x^{2}+\alpha y^{2}+\frac{\alpha \beta-1}{\theta} z^{2}-r^{2} .
$$

Since 0 is a regular value of $g$, then it is well known that $M=g^{-1}(0)$ is a hypersurface in $\mathbb{R}^{3}$. Let $F(x, y, z)=\left(-\alpha y+y z-x^{3}, x-\beta x z-\gamma y^{3}, \theta x y-z^{3}\right)$, $(x, y, z) \in \mathbb{R}^{3}$. Since

$$
\langle\nabla g(x, y, z), F(x, y, z)\rangle=-2 x^{4}-2 \alpha \gamma y^{4}-2 \frac{(\alpha \beta-1)}{\theta} z^{4}<0
$$

for all $(x, y, z) \in M$, then $M$ satisfies condition (T). Thus, by Theorem 9 , the hypersurface $M=g^{-1}(0)$ is an impulsive set which satisfies STC. Note that

$$
\bigcup_{n \in \mathbb{N}}\left\{(x, y, z) \in \mathbb{R}^{3}: x^{2}+\alpha y^{2}+\frac{\alpha \beta-1}{\theta} z^{2}-n^{2}=0\right\}
$$

is also an impulsive set satisfying STC.

Example 12. Let $\left(\mathbb{R}^{n}, \pi\right)$ be a dynamical system given by the system $\dot{x}=f(x)$, where $f \in C^{1}\left(\mathbb{R}^{n}, \mathbb{R}^{n}\right)$ and $x_{i} f_{i}(x)<0$ for all $x \in \mathbb{R}^{n}, x \neq 0$, $i=1,2, \ldots, n$. Let $a_{1}, \ldots, a_{n}, r \in \mathbb{R}_{+} \backslash\{0\}$ and $\alpha_{1}, \ldots, \alpha_{n} \in \mathbb{N}$ be even numbers. Let $M=\left\{\left(x_{1}, \ldots, x_{n}\right) \in \mathbb{R}^{n}: a_{1} x_{1}^{\alpha_{1}}+\ldots+a_{n} x_{n}^{\alpha_{n}}=r\right\}$. If $I: M \rightarrow \mathbb{R}^{n}$ is a continuous map, then $\left(\mathbb{R}^{n}, \pi, M, I\right)$ is an IDS with $M$ satisfying STC.

Example 13. In [2, Example 4.8], the authors analyze the global attractor for the system $\dot{x}=-x, \dot{y}=-y$, in $\mathbb{R}^{2}$ subject to an impulsive function $I: M \rightarrow$ $I(M)$, where $M=\left\{(x, y) \in \mathbb{R}^{2}: x^{2}+y^{2}=1\right\}$. It was just mentioned that $M$ satisfies STC. Using Example 12, we may see easily that $M$ satisfies STC.

\section{Conclusion}

The characterization of impulsive sets that satisfy STC is very important in the theory of impulsive dynamical systems. The function $\phi: X \rightarrow(0,+\infty]$ 
defined in (2) is continuous in $X \backslash M$ when $M$ satisfies STC, see [7, Theorem 3.8]. The continuity of this function is essential to obtain results about invariance, stability, recursive properties, existence of attractors, etc., as the reader can see in [2], [3], [4], [7], [8], [10], [12].

\section{Acknowledgements.}

We would like to thank the anonymous referee for the helpful suggestions which allowed us to improved the presentation of our paper.

\section{References}

[1] E. M. Bonotto. Flows of characteristic $0^{+}$in impulsive semidynamical systems. J. Math. Anal. Appl., 332(1):81-96, 2007.

[2] E. M. Bonotto, M. C. Bortolan, A. N. Carvalho, and R. Czaja. Global attractors for impulsive dynamical systems - a precompact approach. $J$. Differential Equations, 259(7):2602-2625, 2015.

[3] E. M. Bonotto and M. Federson. Topological conjugation and asymptotic stability in impulsive semidynamical systems. J. Math. Anal. Appl., 326(2):869-881, 2007.

[4] E. M. Bonotto and J. C. Ferreira. Uniform attractors of discontinuous semidynamical systems. Collect. Math., 65(1):47-59, 2014.

[5] Bruno Bouchard, Ngoc-Minh Dang, and Charles-Albert Lehalle. Optimal control of trading algorithms: a general impulse control approach. SIAM J. Financial Math., 2(1):404-438, 2011.

[6] Tiziana Cardinali and Raffaella Servadei. Periodic solutions of nonlinear impulsive differential inclusions with constraints. Proc. Amer. Math. Soc., 132(8):2339-2349 (electronic), 2004.

[7] Krzysztof Ciesielski. On semicontinuity in impulsive dynamical systems. Bull. Pol. Acad. Sci. Math., 52(1):71-80, 2004. 
[8] Krzysztof Ciesielski. On stability in impulsive dynamical systems. Bull. Pol. Acad. Sci. Math., 52(1):81-91, 2004.

[9] Mark H. A. Davis, Xin Guo, and Guoliang Wu. Impulse control of multidimensional jump diffusions. SIAM J. Control Optim., 48(8):5276-5293, 2010 .

[10] Changming Ding. Lyapunov quasi-stable trajectories. Fund. Math., 220(2):139-154, 2013.

[11] Morris W. Hirsch. Differential topology. Springer-Verlag, New YorkHeidelberg, 1976. Graduate Texts in Mathematics, No. 33.

[12] Saroop Kaul. On impulsive semidynamical systems. J. Math. Anal. Appl., 150(1):120-128, 1990.

[13] V. Lakshmikantham, D. D. Baĭnov, and P. S. Simeonov. Theory of impulsive differential equations, volume 6 of Series in Modern Applied Mathematics. World Scientific Publishing Co., Inc., Teaneck, NJ, 1989.

[14] Xiaodi Li. Further analysis on uniform stability of impulsive infinite delay differential equations. Appl. Math. Lett., 25(2):133-137, 2012.

[15] Lichun Zhao, Lansun Chen, and Qingling Zhang. The geometrical analysis of a predator-prey model with two state impulses. Math. Biosci., 238(2):55$64,2012$. 